\title{
Socioeconomic factors related to leprosy: an integrative literature review
}

\author{
Fatores socioeconômicos relacionados à hanseníase: revisão integrativa da literatura \\ Factores socioeconómicos relacionados con la lepra: revisión integrativa de la literatura
}

Heloisy Alves de Medeiros Leano'
ORCID: 0000-0001-7337-4079

Kleane Maria da Fonseca Azevedo Araújo' ORCID: 0000-0001-8033-7305

Isabela de Caux Bueno' ORCID: 0000-0003-4501-5989

Eyleen Nabyla Alvarenga Niitsuma ' ORCID: 0000-0002-5781-6313

Francisco Carlos Félix Lana'

ORCID: 0000-0001-9043-3181

'Universidade Federal de Minas Gerais. Belo Horizonte, Minas Gerais, Brazil.

How to cite this article: Leano HAM, Araújo KMFA, Bueno IC, Niitsuma ENA

Lana FCF. Socioeconomic factors related to leprosy: an integrative literature review. Rev Bras Enferm. 2019;72(5):1405-15. doi: http://dx.doi.org/10.1590/0034-7167-2017-0651

\section{Corresponding Author:}

Heloisy Alves de Medeiros Leano E-mail: heloisymedeiros@hotmail.com

Submission: 09-27-2017

Approval: 01-27-2018

\section{ABSTRACT}

Objective: To investigate in the literature the relation of socioeconomic factors in the incidence of the disease and other outcomes related to leprosy. Method: Integrative review conducted in Lilacs, Medline, Scopus databases and SciELO online library with studies from 2000 to 2016. Results: 32 studies were included. Only studies that analyzed statistical associations of socioeconomic factors and outcomes related to leprosy were selected. Conclusion: Leprosy is greatly affected by the social context in which the patient is inserted, the chances of exposure to illness are the result of a set of not only individual aspects, but also of contexts or collective conditions. It is imperative for Nursing, as an essential part of the multiprofessional team entrusted with the care and surveillance of the disease, to recognize these factors to predict unfavorable outcomes and to develop new practices capable of reducing inequities.

Descriptors: Leprosy; Socioeconomic Factors; Public health; Review; Neglected Diseases.

\section{RESUMO}

Objetivo: Investigar na literatura a relação dos fatores socioeconômicos na ocorrência da doença e outros desfechos relacionados à hanseníase. Método: Revisão integrativa realizada nas bases de dados Lilacs, Medline, Scopus e na biblioteca on-line SciELO com estudos de 2000 a 2016. Resultados: Foram incluídos 32 estudos. Apenas pesquisas que analisaram associações estatísticas dos fatores socioeconômicos e os desfechos relacionados à hanseníase foram selecionadas. Conclusão: $\mathrm{A}$ hanseníase sofre grande influência do contexto social em que o doente está inserido, as chances de exposição ao adoecimento são resultantes de um conjunto de aspectos não apenas individuais, mas também de contextos ou condições coletivas. É imperativo à Enfermagem, como parte essencial da equipe multiprofissional incumbida, para o cuidado e vigilância da doença, reconhecer esses fatores para predizer desfechos desfavoráveis e construir novas práticas capazes de reduzir iniquidades.

Descritores: Hanseníase; Fatores Socioeconômicos; Saúde Pública; Revisão; Doenças Negligenciadas.

\section{RESUMEN}

Objetivo: Investigar en la literatura la relación de los factores socioeconómicos en la ocurrencia de la enfermedad y otros resultados relacionados con la lepra. Método: Revisión integrativa realizada en las bases de datos Lilacs, Medline, Scopus y en la biblioteca en línea SciELO con estudios de 2000 a 2016. Resultados: Se incluyeron 32 estudios. Sólo las encuestas que analizaron las asociaciones estadísticas de los factores socioeconómicos y los resultados relacionados con la lepra fueron seleccionados. Conclusión: La lepra sufre una gran influencia del contexto social en que el paciente está inserto, las posibilidades de exposición al enfermo se derivan de un conjunto de aspectos no sólo individuales, sino también de contextos o condiciones colectivas. Es imperativo a la Enfermería, como parte esencial del equipo multiprofesional encargado, para el cuidado y vigilancia de la enfermedad, reconocer esos factores para predecir desenlaces desfavorables y construir nuevas prácticas capaces de reducir iniquidades. Descriptores: Lepra; Factores Socioeconómicos; Salud Pública; Revisión; Enfermedades Desatendidas. 


\section{INTRODUCTION}

Leprosy, despite its declining prevalence in many countries, remains a public health problem through active transmission and discovery of new cases. This is especially true in developing countries that have characteristics that are conducive to the reproduction and spreading of diseases with complex patterns of transmission related to environmental, social, economic and even unknown determinants ${ }^{(1)}$, such as leprosy.

The severity of this disease is present in the physical disabilities resulting from the untreated disease, which can reduce or eliminate opportunities for work and subsistence. In this way, in addition to providing stigma and social isolation, the disease contributes to the reduction of the socioeconomic development of a territory.

Thus, associated with human biology, the social inequalities and inequities present in the space in which people live favor the illness of leprosy and often hinders access to health services and consequently the timely diagnosis and appropriate treatment.

A recent study carried out in Brazil has associated the reduction of the disease burden with the improvement in living conditions, provided by income transfer programs ${ }^{(2)}$. In addition, countries such as Spain and Norway were able to eliminate leprosy even before the appearance of effective medicines against Mycobacterium leprae, and this fact was due to improved living conditions $s^{(3-4)}$.

In this sense, methodological proposals have been used to identify socioeconomic factors of individual and collective scope that favor illness and contribute to complications of leprosy, such as physical disabilities and recurrences.

Nursing professionals play an essential role in the leprosy work process and assume a decisive and proactive role in the planning and execution of care and control actions for patients and contacts $^{(5)}$. Therefore, it can use the results of this study to recognize the influence of social determinants on the healthdisease process, as well as to reflect its practice, aiming to improve leprosy control actions and better understand the socioeconomic factors that are associated with leprosy, in a broader manner in the literature.

\section{OBJECTIVE}

To investigate the relation of socioeconomic factors in the incidence of the disease and other outcomes related to leprosy.

\section{METHOD}

The method used to analyze and synthesize the literature was the integrative review methodology of Whittemore and Knafl(6). There are five phases for the collection, analysis and synthesis of data $^{(6)}$. They are problem identification; literature research; data evaluation; data analysis; and presentation. The initial step was to identify the problem to be addressed, an important time for decision making for data extraction. The problem to be addressed in this review is to identify the socioeconomic factors that are associated with the incidence of leprosy among those who are susceptible and other outcomes among patients. The next four phases are described in the following sections, beginning with literature research.

\section{Literature research}

The survey was conducted in November 2016. The search took place in Lilacs, Medline, Scopus and the SciELO online libraries. The descriptors were selected from the Health Sciences Descriptors (DeCS - decs.bvs.br/) or Medical Subject Headings (Mesh- www.ncbi.nlm.nih.gov/mesh), consolidating the search strategy (Table 1).

Table 1 - Search strategy for each database

\begin{tabular}{|c|c|}
\hline $\begin{array}{l}\text { BVS-ILACS } \\
\text { E MEDLINE }\end{array}$ & $\begin{array}{l}\text { "Social Class" OR "Clase Social" OR "Classe Social" OR } \\
\text { "Socioeconomic Factors" OR"Factores Socioeconómicos" } \\
\text { OR"Fatores Socioeconômicos" OR "poverty" OR"pobreza" } \\
\text { OR"Social Conditions" OR"Condiciones Sociales" OR } \\
\text { "Condições Sociais" OR "Social Indicators" OR"Indicadores } \\
\text { Sociales" OR"Indicadores Sociais") AND (leprosy OR lepra } \\
\text { OR hanseníase) AND (instance:"regional") AND (db: } \\
\text { ("MEDLINE" OR "LILACS") AND ( la:"en"OR"pt"OR"es") } \\
\text { AND (year_cluster:"2012"OR"2011"OR"2013"OR"2010" } \\
\text { OR"2014"OR"2009"OR"2015" OR"2008"OR"2003"OR } \\
\text { "2000") AND ( type:"article"OR"thesis")) }\end{array}$ \\
\hline $\begin{array}{l}\text { PUBME- } \\
\text { MEDLINE }\end{array}$ & $\begin{array}{l}\text { (((("Social Class" [Title/Abstract] OR "Socioeconomic } \\
\text { Factors" [Title/Abstract] OR"Poverty" [Title/Abstract] OR } \\
\text { "Social Conditions" [Title/Abstract] OR "Social Indicators" } \\
\text { [Title/Abstract])) OR (((("Social Class" [Mesh:noexp]) OR } \\
\text { "Socioeconomic Factors" [Mesh:noexp]) OR "Poverty" } \\
\text { [Mesh:noexp]) OR"Social Conditions" [Mesh:noexp]))) AND } \\
\text { (("Leprosy" [Title/Abstract]) OR"Leprosy" [Mesh:noexp]) }\end{array}$ \\
\hline SCOPUS & $\begin{array}{l}\text { ("Social Class" OR "Clase Social" OR "Classe Social" OR } \\
\text { "Socioeconomic Factors" OR "Factores Socioeconómicos" } \\
\text { OR "Fatores Socioeconômicos" OR "poverty" OR } \\
\text { "pobreza" OR "Social Conditions" OR"Condiciones } \\
\text { Sociales" OR "Condições Sociais" OR"Social Indicators") } \\
\text { OR TITLEABSKEY ("Indicadores Sociales" OR "Indicadores } \\
\text { Sociais"))) AND ( TITLEABSKEY ("leprosy" OR"lepra" OR } \\
\text { "hanseníase")) AND (LIMITTO (LANGUAGE, "English" } \\
\text { ) OR LIMITTO (LANGUAGE, "Portuguese") OR LIMITTO } \\
\text { (LANGUAGE, "Spanish")) AND (LIMITTO (PUBYEAR, 2016) } \\
\text { OR LIMITTO (PUBYEAR, 2015) OR LIMITTO (PUBYEAR, } \\
\text { 2014) OR LIMITTO (PUBYEAR, 2013 ) OR LIMITTO } \\
\text { (PUBYEAR, 2012) OR LIMITTO (PUBYEAR, 2011) OR } \\
\text { LIMITTO (PUBYEAR, 2010) OR LIMITTO (PUBYEAR, 2009) } \\
\text { OR LIMITTO (PUBYEAR, 2008) OR LIMITTO (PUBYEAR, } \\
\text { 2007) OR LIMITTO (PUBYEAR, 2006) OR LIMITTO } \\
\text { (PUBYEAR, 2005) OR LIMITTO (PUBYEAR, 2004) OR } \\
\text { LIMITTO (PUBYEAR, 2003) OR LIMITTO (PUBYEAR, 2002) } \\
\text { OR LIMITTO (PUBYEAR, 2001) OR LIMITTO (PUBYEAR, } \\
\text { 2000)) AND (LIMITTO (DOCTYPE, "ar")) }\end{array}$ \\
\hline SCIELO & $\begin{array}{l}\text { ("Social Class" OR "Clase Social" OR "Classe Social" OR } \\
\text { "Socioeconomic Factors" OR"Factores Socioeconómicos" } \\
\text { OR"Fatores Socioeconômicos" OR "poverty" OR "pobreza" } \\
\text { OR "Social Conditions" OR "Condiciones Sociales" OR } \\
\text { "Condições Sociais" OR "Social Indicators" OR "Indicadores } \\
\text { Sociales" OR "Indicadores Sociais") AND ("leprosy" OR } \\
\text { "lepra" OR "hanseníase") }\end{array}$ \\
\hline
\end{tabular}

Publications were extracted from 2000 to 2016, selecting original articles, brief communications, theses and dissertations. The inclusion criteria were presence of abstracts (in Portuguese, English or Spanish) and description of the quantitative approach regarding analysis of statistical association between leprosy and socioeconomic factors. Qualitative and quantitative studies were excluded, with descriptions regarding only frequency measurements. Also excluded were book chapters, event summaries, case reports, editorials and opinion articles. 
Data was searched in the Virtual Health Library Brazil (VHL), with 145 publications in Medline and 45 in Lilacs identified; after that, 15 were found in SciELO, 188 in Scopus, and finally, in Pubmed, 188 articles were identified in Medline, totaling 581 articles. After the removal of duplicates, there were 388 records. After sorting by title and summary, 312 additional records were excluded. Following the application of the inclusion and exclusion criteria, and in full peer reading, 32 articles remained (Figure 1).

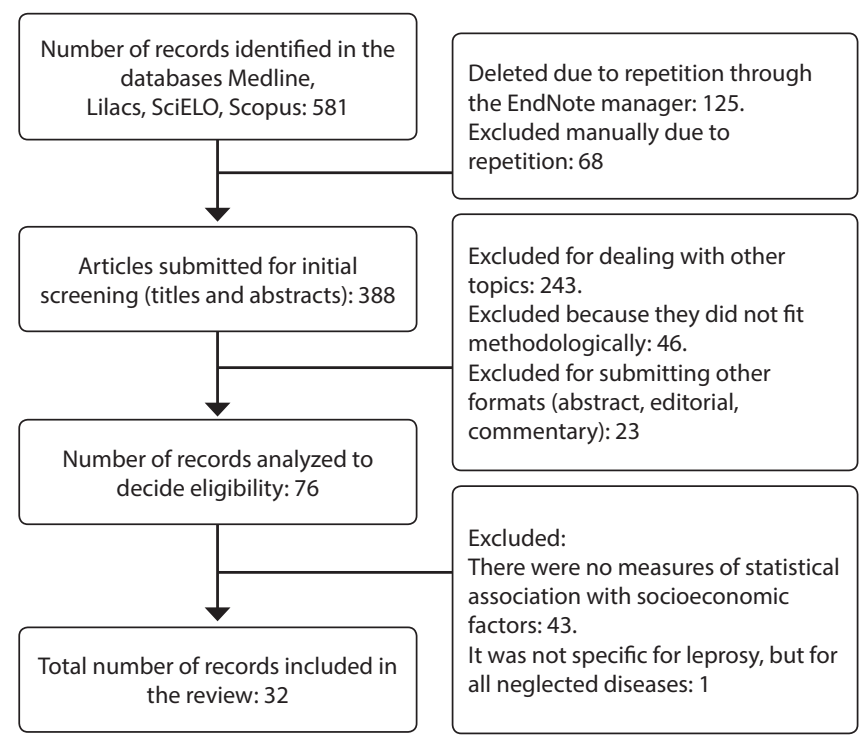

Figure 1 - Diagram of the process of identification, screening, eligibility and inclusion of the studies
For the management of bibliographic references, the online software EndNote (http://www.myendnoteweb.com), made available by Thomson Reuters/Clarivate Analytics, was used.

\section{Assessment and analysis of data}

The next step was the assessment of data. According to Whittemore and $\mathrm{Knafl}^{(6)}$, there are four steps of data analysis: data reduction; data display; comparison of data; and design and verification of conclusions. The 32 articles were initially read for an overview of their response to the research problem. After that, following information was collected: reference, research site, year of study, type of study, sample or aggregate, dependent variable, and socioeconomic factors/indicators associated with the outcome. The studies were discussed in pairs until topics became evident for the formation of categories.

The last stage of the integrative review, the presentation, was displayed in tables in the topic of results, following below.

\section{RESULTS}

Of the 22 studies that analyzed the socioeconomic factors associated with the incidence of the disease and other leprosyrelated outcomes at the individual level, seven were case-control studies, four were cut-offs, and eleven were cross-sectional (Chart 1). Six categories were extracted from the results presented.

At the ecological level, of the ten studies selected for this review, five sought association with municipalities as a unit of analysis, one with micro-regions and four using the census divisions (Chart 2). Of these, two categories were compiled.

Chart 1 - Individual-level studies on the association between socioeconomic factors and leprosy

\begin{tabular}{|c|c|c|c|c|c|c|}
\hline References & Country & Year & $\begin{array}{l}\text { Type of } \\
\text { Study }\end{array}$ & Sample & $\begin{array}{c}\text { Dependent } \\
\text { variable }\end{array}$ & Associated indicators \\
\hline $\begin{array}{l}\text { DIFFEY et } \\
\mathrm{al}^{2} 2000^{(7)} \text {. }\end{array}$ & India & 1997 & $\begin{array}{c}\text { Cross- } \\
\text { sectional }\end{array}$ & $\begin{array}{l}871 \text { subjects, } \\
\text { of these, } 155 \\
\text { cases with } \\
\text { deformity, } \\
100 \text { cases } \\
\text { without } \\
\text { deformities } \\
\text { and } 616 \\
\text { household } \\
\text { contacts. }\end{array}$ & $\begin{array}{l}\text { Presence } \\
\text { or absence } \\
\text { of physical } \\
\text { impairment. }\end{array}$ & $\begin{array}{l}\text { Age of the index case (test } t p<0.0005) \text {, number of wage } \\
\text { earners (test } t p<0.05) \text {, income from inside houses contacts } \\
\text { (test } t p<0.01) \text {, weekly expenses on food (test } t p<0.05 \text { ), } \\
\text { proportion of deformity in the lowest socioeconomic classes } \\
(4 \text { and } 5)\left(x^{2} p<0.01\right) \text {, proportion of male contacts in lower } \\
\text { socioeconomic classes }\left(x^{2} p<0.006\right) \text {, educational level among } \\
\text { male cases }\left(x^{2} p<0.002\right) \text {, illiteracy among women }\left(x^{2} p<0.001\right) \text {, } \\
\text { wage among sex }(p<0.01) \text {, and unemployment among groups } \\
(p<0.0005) \text {. }\end{array}$ \\
\hline $\begin{array}{l}\text { FERREIRA; } \\
\text { IGNOTTI.; } \\
\text { GAMBA; } \\
2011^{(8)}\end{array}$ & Brazil. & $\begin{array}{c}2005- \\
2007\end{array}$ & $\begin{array}{l}\text { Case } \\
\text { control }\end{array}$ & $\begin{array}{l}53 \text { cases with } \\
\text { recurrence } \\
\text { from } 2005 \text { to } \\
2007 \text {. } \\
106 \text { controls } \\
\text { with } \\
\text { discharge for } \\
\text { cure in } 2005 \text {. }\end{array}$ & $\begin{array}{l}\text { Recurrence } \\
\text { cases }\end{array}$ & 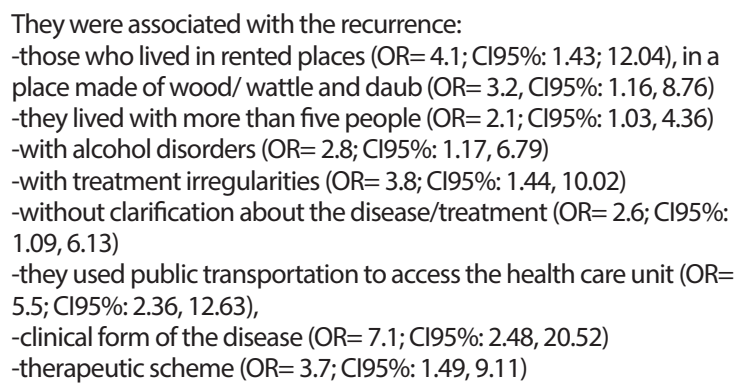 \\
\hline $\begin{array}{l}\text { FABRI, } \\
2011^{(9)} \text {. }\end{array}$ & Brazil & $\begin{array}{l}2010- \\
2011\end{array}$ & $\begin{array}{c}\text { Cross- } \\
\text { sectional }\end{array}$ & 2,726 people & Seropositivity & $\begin{array}{l}\text {-Family income } \\
<1 \text { minimum wage }(p=0.035, C \mid 95 \%, 1.06-4.86) \\
\text {-Number of people living in the house } \\
4-7(p=0.04, C \mid 95 \%, 0.07-0.94) \geq 8(p=0.075, C \mid 95 \%, 0.09-1.12) \\
\text {-Number of rooms in the house } \\
1-5(p=0.122, C \mid 95 \%, 0.85-3.84)\end{array}$ \\
\hline
\end{tabular}


Chart 1

\begin{tabular}{|c|c|c|c|c|c|c|}
\hline References & Country & Year & $\begin{array}{l}\text { Type of } \\
\text { Study }\end{array}$ & Sample & $\begin{array}{l}\text { Dependent } \\
\text { variable }\end{array}$ & Associated indicators \\
\hline $\begin{array}{l}\text { HEGAZY et } \\
\text { al., } 2002^{(10)}\end{array}$ & Egypt & $\begin{array}{l}1999- \\
2001\end{array}$ & $\begin{array}{l}\text { Case } \\
\text { control }\end{array}$ & $\begin{array}{l}24 \text { Cases of } \\
\text { leprosy, } 124 \\
\text { contacts inside } \\
\text { the house, } \\
30 \text { contacts } \\
\text { outside the } \\
\text { house }\end{array}$ & Incidence & $\begin{array}{l}\text { Prevalence of leprosy among illiterates }(\mathrm{OR}=3.69, \mathrm{Cl}= \\
0.83-23.02, \mathrm{p}=0.103) ; \text { regarding elementary education }(\mathrm{OR}= \\
1.41, \mathrm{Cl}=0.22-11.09, \mathrm{p}=1) ; \text { more than } 4 \text { people sharing a room } \\
(\mathrm{OR}=1.3, \mathrm{Cl}=0.49-3.33, \mathrm{p}=0.556) \text {, hand pump water supply } \\
(\mathrm{OR}=1.39, \mathrm{Cl}=0.56-3.54, \mathrm{p}=0.444) \text {, lower class }(\mathrm{OR}=2.43, \mathrm{Cl}= \\
0.86-7.44, \mathrm{p}=0.067)\end{array}$ \\
\hline $\begin{array}{l}\text { NARDI et } \\
\text { al., } 2012^{(11)} \text {. }\end{array}$ & Brazil & $\begin{array}{l}1998- \\
2006\end{array}$ & $\begin{array}{l}\text { Cross- } \\
\text { sectional }\end{array}$ & $\begin{array}{l}335 \text { patients } \\
\text { treated }\end{array}$ & $\begin{array}{l}\text { Physical } \\
\text { impairment }\end{array}$ & $\begin{array}{l}\text { Average of people who are of age }\left(x^{2} p=0.029\right) \text {, education }\left(x^{2}\right. \\
p=0.051)\end{array}$ \\
\hline $\begin{array}{l}\text { ARAUJO et } \\
\text { al., } 2014^{(12)} \text {. }\end{array}$ & Brazil & $\begin{array}{l}2010- \\
2011\end{array}$ & $\begin{array}{l}\text { Cross- } \\
\text { sectional }\end{array}$ & 155 cases & $\begin{array}{l}\text { Neural changes } \\
\text { and physical } \\
\text { impairment }\end{array}$ & $\begin{array}{l}\text { Education: } \\
\text {-No education OR } 1 \\
\text {-Low education levels OR } 0.75(p=0.81,0.07-8.09) \\
\text {-Average education levels OR } 1.78(p=0.30,0.60-5.27) \\
\text {-High education levels OR } 1.23(p=0.68,0.43-3.56) \\
\text { Family income (minimum wage) } \\
\text { - >3 OR } 1 ;>1-2 \text { OR } 0.57(p=0.156,0.26-1.24) ;<1 \text { OR } 1.20(p=0.729, \\
0.42-3.45)\end{array}$ \\
\hline $\begin{array}{l}\text { KERR- } \\
\text { PONTES et } \\
\text { al., } 2006^{(13)}\end{array}$ & Brazil & 2002 & $\begin{array}{l}\text { Case } \\
\text { control }\end{array}$ & $\begin{array}{l}200 \text { cases and } \\
800 \text { control }\end{array}$ & Incidence & $\begin{array}{l}\text { High School }(\mathrm{OR}=1.50, \mathrm{Cl}=0.91-2.50) \text {, lower education level } \\
(\mathrm{OR}=1.87, \mathrm{Cl}=1.29-2.74) \text {, having experienced food shortages } \\
(\mathrm{OR}=1.54, \mathrm{Cl}=1.45-1.63) .\end{array}$ \\
\hline $\begin{array}{l}\text { MURTO et } \\
\text { al., } 2014^{(14)} \text {. }\end{array}$ & Brazil & 2009 & $\begin{array}{l}\text { Cross- } \\
\text { sectional }\end{array}$ & $\begin{array}{l}1,074 \text { cases of } \\
\text { leprosy }\end{array}$ & $\begin{array}{l}\text { Migration } \\
\text { status }\end{array}$ & 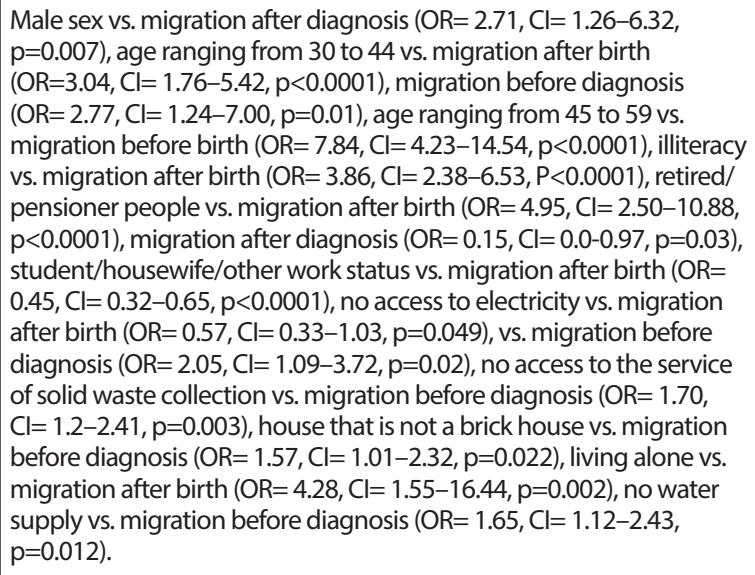 \\
\hline $\begin{array}{l}\text { SANTOS et } \\
\text { al., } 2013^{(15)} \text {. }\end{array}$ & Brazil & $\begin{array}{l}1987- \\
2010\end{array}$ & $\begin{array}{c}\text { Prospective } \\
\text { cut }\end{array}$ & $\begin{array}{l}\text { 7,174 contacts } \\
\text { (incidence) } \\
7,012 \\
\text { (prevalence) }\end{array}$ & $\begin{array}{l}\text { Prevalence } \\
\text { among } \\
\text { contacts }\end{array}$ & $\begin{array}{l}\text { Education } \\
\text { (prevalence) } \\
\text { >10 years OR } 1 ; 4 \text { to } 10 \text { years OR } 1.33(0.81-2.18) ;<4 \text { years OR } 2.18 \\
(1.42-3.35) \\
\text { Skin color } \\
\text { (prevalence): White OR 1; Brown/Black OR 1.32 }(1.02-1.70) \\
\text { (incidence): White OR 1; Brown/Black OR 1.66 }(1.14-2.42)\end{array}$ \\
\hline $\begin{array}{l}\text { SAMUEL et } \\
\text { al., 2012(16). }\end{array}$ & India & $\begin{array}{l}2004- \\
2008\end{array}$ & $\begin{array}{l}\text { Cross- } \\
\text { sectional } \\
\text { survey }\end{array}$ & $\begin{array}{l}222 \text { cases of } \\
\text { leprosy were } \\
\text { not treated }\end{array}$ & Migration & $\begin{array}{l}\text { Age adult vs. child }\left(x^{2}=1.635, p=0.200\right) \text {, married } v s \text {. single }\left(x^{2}=\right. \\
1.588, p=0.207) \text {, education illiterate people vs. people with some } \\
\text { level of education }\left(x^{2}=0.024, p=0.961\right)\end{array}$ \\
\hline $\begin{array}{l}\text { FEENSTRA } \\
\text { et al., } \\
2011^{(17)}\end{array}$ & Bangladesh & 2009 & $\begin{array}{l}\text { Case } \\
\text { control }\end{array}$ & $\begin{array}{l}99 \text { patients } \\
\text { (cases) and } \\
199 \text { controls }\end{array}$ & $\begin{array}{l}\text { Manifestation } \\
\text { of leprosy }\end{array}$ & $\begin{array}{l}\text { A recent period of food shortage was identified as the only } \\
\text { socioeconomic factor significantly associated with the clinical } \\
\text { manifestation of leprosy, not poverty itself (OR } 1.79(1.06-3.02) \text {, } \\
p=0.030) \text {. There is a declining trend in the prevalence of leprosy } \\
\text { with a growing socioeconomic status, measured with an active } \\
\text { index, but not statistically significant (test for a trend: OR } 0.85 \\
(0.71-1.02) p=0.083) \text {. }\end{array}$ \\
\hline $\begin{array}{l}\text { HEUKEL- } \\
\text { BACH, et al., } \\
2011^{(18)} \text {. }\end{array}$ & Brazil & 2009 & $\begin{array}{l}\text { Cross- } \\
\text { sectional } \\
\text { population } \\
\text { study }\end{array}$ & $\begin{array}{l}936 \text { people/ } \\
\text { patients }\end{array}$ & $\begin{array}{l}\text { Treatment } \\
\text { interruption }\end{array}$ & $\begin{array}{l}2.42(1.02-5.63) \text { - Family income/month less than } 465 \text { Brazilian } \\
\text { reais }\end{array}$ \\
\hline
\end{tabular}


Chart 1 (concluded)

\begin{tabular}{|c|c|c|c|c|c|c|}
\hline References & Country & Year & $\begin{array}{l}\text { Type of } \\
\text { Study }\end{array}$ & Sample & $\begin{array}{c}\text { Dependent } \\
\text { variable }\end{array}$ & Associated indicators \\
\hline $\begin{array}{l}\text { RIBEIRO, } \\
2012^{(19)} .\end{array}$ & Brazil & $\begin{array}{l}2005- \\
2010\end{array}$ & $\begin{array}{c}\text { Prospective } \\
\text { cut }\end{array}$ & $\begin{array}{l}71 \\
\text { participants }\end{array}$ & $\begin{array}{l}\text { Level of } \\
\text { physical } \\
\text { impairment in } \\
\text { diagnosis }\end{array}$ & $\begin{array}{l}\text { In the bivariate analysis there was a statistical relation between } \\
\text { education }(p=0.032) \\
\text { Education } P=0.031^{* *} \text { Fisher's test } \\
\text { Family income } P=0.860 \\
\text { Number of people in the house } P=0.267 \\
\text { Occupation } P=0.835 \\
\text { Current occupation } P=0.122\end{array}$ \\
\hline $\begin{array}{l}\text { SANTOS; } \\
\text { CASTRO; } \\
\text { FALQUETO, } \\
2008^{(20)}\end{array}$ & Brazil & $\begin{array}{l}2003- \\
2006\end{array}$ & $\begin{array}{c}\text { Case } \\
\text { control }\end{array}$ & $\begin{array}{l}90 \text { cases } \\
270 \text { controls }\end{array}$ & $\begin{array}{l}\text { Incidence of } \\
\text { the disease }\end{array}$ & $\begin{array}{l}\text { Education: Elementary school OR: } 1.092 \text { (0.616-1.937); High } \\
\text { school OR: } 1.455(0.684-3.096) ; \text { Undergraduate school OR: } 1.682 \\
\text { (0.289-9.804) } \\
\text { Income: Between } 1 \text { and } 3 \text { OR: } 0.698(0.393-1.240) \text {; Greater than } \\
\text { 3 OR: } 1,070(0,360-3,179)\end{array}$ \\
\hline $\begin{array}{l}\text { WAGE- } \\
\text { NAAR et al., } \\
2015^{(21)}\end{array}$ & Bangladesh & 2013 & $\begin{array}{l}\text { Case } \\
\text { control }\end{array}$ & $\begin{array}{l}52 \text { cases } \\
100 \text { controls }\end{array}$ & $\begin{array}{l}\text { Incidence of } \\
\text { the disease }\end{array}$ & $\begin{array}{l}\text { Having higher food expenses per capita(log) (OR: } 0.03-\mathrm{Cl} 0.00- \\
0.36) \text {, being a farmer (OR: } 0.24 \mathrm{Cl}: 0.07,0.83) \text { and owning a business } \\
\text { (OR: } 0.31 \mathrm{Cl}: 0.07-1.34) \text { were protection factors }\end{array}$ \\
\hline $\begin{array}{l}\text { MONDAL et } \\
\text { al., } 2015^{(22)} \text {. }\end{array}$ & India & 2015 & Cut & $\begin{array}{l}50 \text { patients } \\
\text { with leprosy }\end{array}$ & $\begin{array}{l}\text { Prevalence of } \\
\text { reactions }\end{array}$ & $\begin{array}{l}\text { Living in rural areas }\left(x^{2}, p=0.9345\right) \text {, socioeconomic condition }\left(x^{2} \text {, }\right. \\
p=0.6137) \text {, education }\left(x^{2}, p=0.7842\right)\end{array}$ \\
\hline $\begin{array}{l}\text { SANYAL, et } \\
\text { al., } 2011^{(23)} \text {. }\end{array}$ & India & $\begin{array}{c}2001- \\
2002\end{array}$ & Survey & $\begin{array}{l}93 \text { patients } \\
\text { with leprosy }\end{array}$ & $\begin{array}{l}\text { Social harm and } \\
\text { mental illness }\end{array}$ & $\begin{array}{l}\text { Impairment present vs. living in urban areas vs. rural areas }\left(x^{2}=\right. \\
5.455 \text {, adjusted } p=0.02) \text {. }\end{array}$ \\
\hline $\begin{array}{l}\text { COSTA et al., } \\
2012^{(24)}\end{array}$ & Brazil & $\begin{array}{c}2007 \text { to } \\
2008\end{array}$ & $\begin{array}{l}\text { Cross- } \\
\text { sectional }\end{array}$ & 120 patients & $\begin{array}{l}\text { Quality of life } \\
\text { of patients } \\
\text { with leprosy } \\
\text { reactions }\end{array}$ & $\begin{array}{l}\text { People married }(p=0.001) \text {, education level }(p>0.05) \text {, occupation } \\
\text { and quality of life regarding physical, psychological and } \\
\text { environmental aspects }(p<0.05) \text {, interference of the disease in } \\
\text { occupation }(p<0.001) \text {, interference in the professional activity } \\
\text { and family income }(p<0.001) \text {, family income and physical aspect } \\
\text { ( } p=0.001) \text {, family income and psychological aspect }(p=0.026) \text {, } \\
\text { family income and environmental aspect }(p=0.025)\end{array}$ \\
\hline $\begin{array}{l}\text { KAR; PAL; } \\
\text { BHARATI, } \\
2010^{(25)}\end{array}$ & India & $\begin{array}{c}2002 \text { to } \\
2005\end{array}$ & $\begin{array}{l}\text { Retrograde } \\
\text { cut }\end{array}$ & $\begin{array}{l}1,020 \text { cases of } \\
\text { leprosy }\end{array}$ & $\begin{array}{l}\text { Adherence to } \\
\text { pharmacological } \\
\text { therapy }\end{array}$ & $\begin{array}{l}\text { Male sex }\left(x^{2}=5.873, p=0.0154\right) \text {, education level }\left(x^{2}=32.350,\right. \\
p<0.0001) \text {, monthly income per capita }\left(x^{2}=22.150, p=0.0005\right) \text {, } \\
\text { socioeconomic status }\left(x^{2}=66.735, p<0.0001\right)\end{array}$ \\
\hline $\begin{array}{l}\text { KUMAR et } \\
\text { al., } 2004^{(26)} \text {. }\end{array}$ & Nepal & $\begin{array}{c}2001 \text { to } \\
2003\end{array}$ & $\begin{array}{l}\text { Cross- } \\
\text { sectional }\end{array}$ & $\begin{array}{l}580 \text { patients } \\
\text { with leprosy }\end{array}$ & $\begin{array}{l}\text { Adherence to } \\
\text { treatment }\end{array}$ & $\begin{array}{l}\text { Sex }(\mathrm{OR}=2.05, \mathrm{Cl}=1.07-3.94) \text {, Age }(\mathrm{OR}=1.76, \mathrm{Cl}=0.81-3.80) \text {, } \\
\text { Education status (literate vs. illiterate) }(\mathrm{OR}=2.37, \mathrm{Cl}=1.12-4.99) \text {, Caste } \\
\text { level }(\mathrm{OR}=1.23, \mathrm{Cl}=0.40-3.74) \text {, Religion }(\mathrm{OR}=0.45, \mathrm{Cl}=0.14-1.43) \text {, } \\
\text { Occupation: farmer vs. unemployed }(\mathrm{OR}=1.29, \mathrm{Cl}=0.49-3.40) \text {, } \\
\text { employed vs. unemployed }(\mathrm{OR}=0.42 ; \mathrm{Cl}=0.14-1.20) \text {, business/ } \\
\text { service vs. unemployed ( } \mathrm{OR}=1.00, \mathrm{Cl}=0.27-3.70), \text { Family type }(\mathrm{OR}= \\
0.75, \mathrm{Cl}=0.38-1.45), \text { Annual family income }(\mathrm{OR}=1.18, \mathrm{Cl}=0.29-4.75) \text {, } \\
\text { Hectares of land belonging to the family }(\mathrm{OR}=0.60, \mathrm{Cl}=0.29-1.23) \text {. }\end{array}$ \\
\hline $\begin{array}{l}\text { MURTO et } \\
\text { al., } 2013^{(27)} \text {. }\end{array}$ & Brazil & 2010 & $\begin{array}{c}\text { Case } \\
\text { control }\end{array}$ & $\begin{array}{l}394 \text { cases and } \\
391 \text { controls }\end{array}$ & Incidence & $\begin{array}{l}\text { Monthly income less than } 1 \text { minimum wage (OR: } 2.12, \mathrm{Cl}: 0.97-4.71 \text {, } \\
p=0.049) \text {, little access to public cleaning services (OR: } 3.1, \mathrm{Cl}: 1.1- \\
10.02, p=0.03) \text {, illiteracy in family (OR: } 2.67, \mathrm{Cl}: 1.13-6.51, \mathrm{p}=0.02)\end{array}$ \\
\hline $\begin{array}{l}\text { WITHINGTON } \\
\text { etal., } 2003^{(28)} \text {. }\end{array}$ & India & 1996 & Cut & $\begin{array}{l}2,364 \text { new } \\
\text { cases of } \\
\text { leprosy }\end{array}$ & $\begin{array}{l}\text { Physical } \\
\text { impairment } \\
\text { and stigma }\end{array}$ & 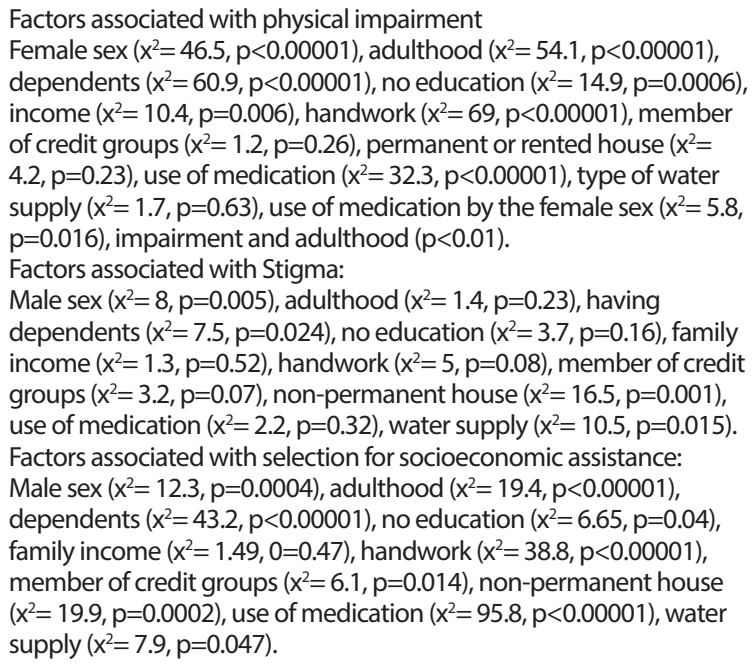 \\
\hline
\end{tabular}


Chart 2 - Environmental-level studies on the association between socioeconomic factors and leprosy

\begin{tabular}{|c|c|c|c|c|c|c|}
\hline Reference & Country & Year & Type of study & $\begin{array}{c}\text { Damage } \\
\text { aggregation }\end{array}$ & $\begin{array}{l}\text { Dependent } \\
\text { variable }\end{array}$ & Associated indicators \\
\hline $\begin{array}{l}\text { AMARAL, } \\
2008^{(29)}\end{array}$ & Brazil & $\begin{array}{l}1998 \\
\text { to } \\
2006\end{array}$ & $\begin{array}{l}\text { Ecological, } \\
\text { retrospective }\end{array}$ & Census Tracts & $\begin{array}{l}\text { Average of } \\
\text { detection } \\
\text { rate }\end{array}$ & $\begin{array}{l}\text { The use of the Kruskal-Wallis test showed that the average } \\
\text { of leprosy detection coefficients in the Low Risk category } \\
\text { is higher than in the other categories, and that the average } \\
\text { of Medium Risk category is higher than that of High-Risk } \\
\text { category and Very High ( } p<0.05) \text {. There were no statistically } \\
\text { significant differences between the averages of the last two } \\
\text { categories. }\end{array}$ \\
\hline $\begin{array}{l}\text { KERR- } \\
\text { PONTES et } \\
\text { al., } 2004^{(30)} \text {. }\end{array}$ & Brazil & $\begin{array}{l}1991 \\
\text { to } \\
1996\end{array}$ & Ecological & 165 Cities & Incidence & $\begin{array}{l}\text { Inequality level }- \text { Theil index }(\beta=1.67, C l=0.389-2.944 \text {, } \\
p=0.011) \text {, average years of study of the population } \geq 25 \\
\text { years }(\beta=1.35, C l=0.620-2.081, p=0.007) \text {, population } \\
\text { growth from } 1991 \text { to } 1996(\beta=0.02, C l=0.006,0.038 \\
p=0.028) \text {. }\end{array}$ \\
\hline $\begin{array}{l}\text { QUEIROZ et } \\
\text { al., 2010(31). }\end{array}$ & Brazil & $\begin{array}{l}1995 \\
\text { to } \\
2006\end{array}$ & Ecological & Census Tract & $\begin{array}{l}\text { Cases of } \\
\text { leprosy }\end{array}$ & $\begin{array}{l}\text { Factor } 1 \text { (basic sanitation - existence of piped water, } \\
\text { presence of toilets in the house and collection of garbage) } \\
\beta=0.0978, p=0.0331 \\
\text { Factor } 2 \text { (level of literacy and income - average family } \\
\text { income, years of education and number of bathrooms in } \\
\text { the house) did not associate. } \\
\text { Factor } 3 \text { (poverty level - lack of access to bank loans, and the } \\
\text { number of people living in a house) } \beta=0.01027, p=0.0240\end{array}$ \\
\hline $\begin{array}{l}\text { LANA et al., } \\
2009^{(32)} \text {. }\end{array}$ & Brazil & $\begin{array}{l}2003 \\
\text { to } \\
2006\end{array}$ & Cross-sectional & City & $\begin{array}{l}\text { Detection } \\
\text { rate }\end{array}$ & Low HDI $(p=0.002)$ \\
\hline $\begin{array}{l}\text { NERY et al., } \\
2014^{(2)} \text {. }\end{array}$ & Brazil & $\begin{array}{l}2004 \\
\text { to } \\
2011\end{array}$ & Ecological & Cities & $\begin{array}{l}\text { Detection } \\
\text { rate of } \\
\text { leprosy }\end{array}$ & 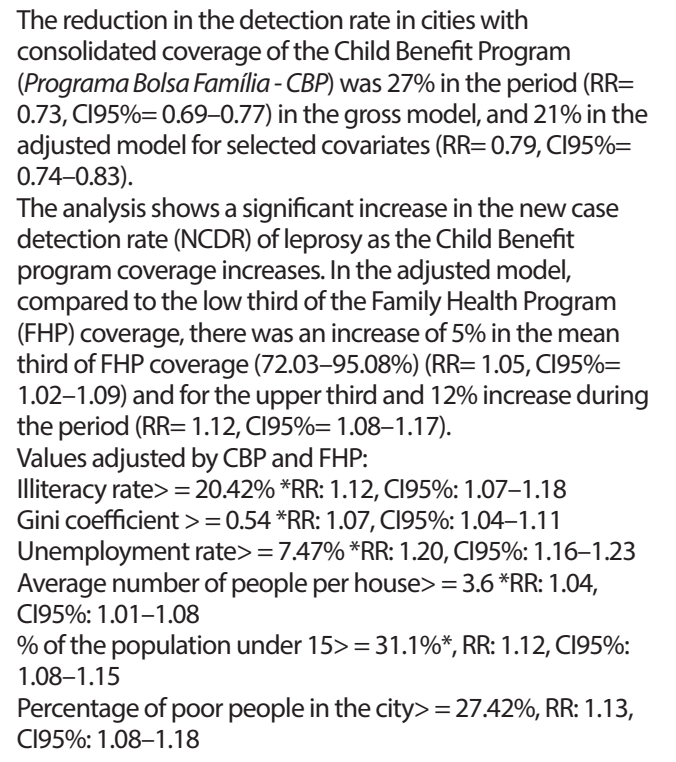 \\
\hline $\begin{array}{l}\text { CURY et al., } \\
2012^{(33)} \text {. }\end{array}$ & Brazil & $\begin{array}{c}1998 \text { to } \\
2007\end{array}$ & Ecological & $\begin{array}{l}\text { Census tracts of } \\
\text { São José do Rio } \\
\text { Preto City }\end{array}$ & $\begin{array}{l}\text { Spatial } \\
\text { distribution } \\
\text { of leprosy } \\
\text { incidence } \\
\text { per } 100,000 \\
\text { inhab. }\end{array}$ & $\begin{array}{l}\text { Index composed of socioeconomic conditions: average } \\
\text { years of study of the father and mother, average income } \\
\text { of the father and mother, percentage of illiterate people, } \\
\text { percentage of illiterate women and percentage of houses } \\
\text { with } 5 \text { or more residents }\left(x^{2}=180.7 ; p<0.0001\right)\end{array}$ \\
\hline $\begin{array}{l}\text { IMBIRIBA et al., } \\
2009^{(34)} \text {. }\end{array}$ & Brazil & $\begin{array}{c}1998 \text { to } \\
2004\end{array}$ & Ecological & $\begin{array}{l}\text { Census tracts of } \\
\text { Manaus City }\end{array}$ & $\begin{array}{l}\text { Detection } \\
\text { rate of } \\
\text { leprosy }\end{array}$ & $\begin{array}{l}\text { Social deprivation index (ICS) composed of variables: } \\
\text { number of people per house, houses without a toilet, } \\
\text { without a sewage system, without water supply, illiterate } \\
\text { head of household, no education or less than } 1 \text { year } \\
\text { of study, monthly income of up to } 1 \text { minimum wage, } \\
\text { head of household with no monthly income, years } \\
\text { of education of the head of household and average } \\
\text { monthly income. Average life expectancy (OR=1.665, } \\
\mathrm{Cl}=1.136-2.441, \mathrm{p}=0.009) \text {, average to low life expectancy } \\
\text { (OR=3.048, } \mathrm{Cl}=2.152-4.317, \mathrm{p}=0.000), \text { low life } \\
\text { expectancy }(\mathrm{OR}=4.427, \mathrm{Cl}=3.140-6.242, \mathrm{p}=0.000) \text {. }\end{array}$ \\
\hline
\end{tabular}




\begin{tabular}{|c|c|c|c|c|c|c|}
\hline Reference & Country & Year & Type of study & $\begin{array}{c}\text { Damage } \\
\text { aggregation }\end{array}$ & $\begin{array}{c}\text { Dependent } \\
\text { variable }\end{array}$ & Associated indicators \\
\hline $\begin{array}{l}\text { FREITAS; } \\
\text { DUARTE; } \\
\text { GARCIA; } \\
2014^{(35)}\end{array}$ & Brazil & $\begin{array}{l}2009 \text { to } \\
2011\end{array}$ & Ecological & City & $\begin{array}{l}\text { Average } \\
\text { smoothed } \\
\text { incidence } \\
\text { rate }\end{array}$ & 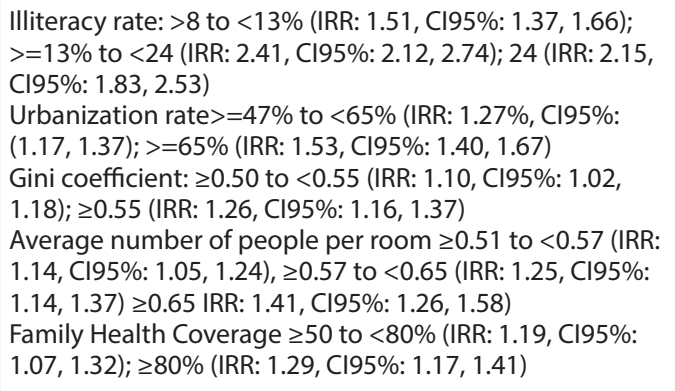 \\
\hline $\begin{array}{l}\text { SILVA et al., } \\
2010^{(36)} \text {. }\end{array}$ & Brazil & 2006 & Ecological & $\begin{array}{l}105 \\
\text { microregions } \\
\text { of the Brazilian } \\
\text { Amazon forest }\end{array}$ & $\begin{array}{l}\text { Detection } \\
\text { rate of } \\
\text { leprosy }\end{array}$ & $\begin{array}{l}\text { Proportion of people in houses with rudimentary cesspits } \\
\text { (ANOVA Average }=3.979, \mathrm{p}=0.000 \text { ), } \mathrm{HDI} \text { year } 2000 \\
\text { (ANOVA Average }=0.233, \mathrm{p}=0.000 \text { ), proportion of people } \\
\text { living in houses with well-water supply (ANOVA Average= } \\
0.056, \mathrm{p}=0.880 \text { ) }\end{array}$ \\
\hline \begin{tabular}{l|} 
CABRAL- \\
MIRANDA; \\
CHIARAVALLOTI \\
NETO; \\
BARROZO, \\
2014 \\
\end{tabular} & Brazil & $\begin{array}{c}2005 \text { to } \\
2011\end{array}$ & Ecological study & Cities & $\begin{array}{l}\text { Detection } \\
\text { rate of } \\
\text { leprosy in } \\
\text { children } \\
\text { under } 15\end{array}$ & $\begin{array}{l}>\text { average number of inhabitants per city }(\beta=0.43 \text {, } \\
p=0.04) \text {; Gini coefficient }(\beta=3.84, p<0.001) ; \% \text { of urban } \\
\text { population }(\beta=0.02, p<0.001) ; \% \text { of population born in } \\
\text { Bahia State }(\beta=-0.04, p<0.001) \text {. }\end{array}$ \\
\hline
\end{tabular}

It is important to highlight that, although there was a study with measures of association between leprosy reactions and socioeconomic factors ${ }^{(22)}$, a significant statistical association was not demonstrated and was not considered among the categories.

\section{Categories for studies at the individual level}

\section{Socioeconomic factors related to the incidence of leprosy}

In relation to incident cases of leprosy, all studies presented a case-control study (Brazil, Egypt and Bangladesh) and showed a positive association for low educational level ${ }^{(13)}$, experience of food shortage ${ }^{(13,17)}$, whereas having higher expenses with food, being a farmer and owner of the business, itself was a protective effect ${ }^{(21)}$. Other studies did not show the association of this disease with socioeconomic factors ${ }^{(10,20)}$.

\section{Socioeconomic factors related to the incidence of physical impairment}

As for the factors associated with impairment, studies indicated an association with low or no schooling ${ }^{(7,10-11,19,28)}$ and low family income ${ }^{(7,28)}$. Only one study did not show any association with socioeconomic variables ${ }^{(12)}$.

Migration was also found as an important factor for the maintenance of the disease chain, demonstrating among migrants an increased risk of illness due to the social or household contact of a patient, alcohol user, not having access to public services of waste collection $^{(27)}$ and being illiterate ${ }^{(16,27)}$. For migration after diagnosis, we observed an association with the male, retired or pensioner, who migrated five years before diagnosis, from zero to five years living in the current residence. For the migration before diagnosis the age range from 30 to 44 years was associated, as well as not having access to electricity, not living in a brick-made house, being migrant after diagnosis, and living in the current residence from zero to five years or six to ten years ${ }^{(14)}$.

\section{Socioeconomic factors related to the incidence of leprosy among inside and outside the person's house}

Regarding the incidence and prevalence of leprosy, among inside and outside the person's house contacts, of the cases diagnosed in a referral service ${ }^{(15)}$, the prevalence was associated with black/brown skin color; years of study less than four, living in the same house, having more than five years of living with the index case, and presenting bacterial index $>3$. The incidence was also associated with black/brown color, bacterial index $>0$; and the BCG vaccine showed a protective effect on both the incidence and the prevalence of leprosy among the contacts. For the contacts, there was an association with seropositivity for the PGL1 antigen among those with a family income below a minimum wage and the number of residents ranging from four to seven people ${ }^{(9)}$.

\section{Socioeconomic factors related to adherence to treatment}

Regarding adherence to the polychemotherapy treatment, there was an association between discontinuation of treatment for males and low educational level ${ }^{(25-26)}$, in addition to the higher risk of abandonment among those with low per capita income ${ }^{(25)}$ and income less than 465 Brazilian reais ${ }^{(18)}$.

\section{Socioeconomic factors related to recurrence}

Regarding recurrence, a control case study found a greater chance among those living in rented houses, with more than five people, houses made of wood or wattle and daub, with alcoholrelated disorders, presenting an irregularity in the treatment, no 
information about the disease, and using collective transportation for access to the health unit ${ }^{(8)}$.

\section{Socioeconomic factors related to the quality of life of patients with leprosy}

As for quality of life, better results were shown among married patients. Retired people, with lower family income, and having reported disease interference in professional activities had a worse score ${ }^{(24)}$. When there is physical impairment, the social and mental harm to those living in rural areas is higher ${ }^{(23)}$.

\section{Categories for studies at the ecological level}

\section{Socioeconomic factors related to the detection rate in aggregates}

A relation of higher incidence of cases with worse Human Development Index (HDI) ${ }^{(32,36)}$ was shown. The studies that sought to analyze the incidence of leprosy and socioeconomic factors, having as a level of aggregation the municipalities, observed an association with the following variables: The Theil index, average of study of population $\geq 25$ years, percentage of children who are between seven and fourteen years old and do not go to school(30); the Gini coefficient, education, illiteracy rate $\mathrm{e}^{(2,35)}$; rate of urbanization, average number of inhabitants per room ${ }^{(35)}$; number of residents per house, unemployment rate and percentage of poor people ${ }^{(2)}$.

The coverage of the Child Benefit program is inversely associated with detection, and its increase is attributed to a decrease in new cases of leprosy ${ }^{(2)}$.

Investigations on the relationship between socioeconomic factors and the detection of leprosy, using the census tracts as a spatial aggregation of data, pointed to a direct association with composite indicators ${ }^{(29,33-34)}$. Another study made a correlation analysis and factorial load of variables. Three factors were extracted for analysis, and two of them were associated: basic sanitation and poverty rate ${ }^{(31)}$.

A study conducted by Silva ${ }^{(36)}$ in micro regions of the Amazon forest showed an association between the rate of detection of the disease and a higher proportion of houses with a rudimentary cesspit and well-water supply.

\section{Socioeconomic factors related to the detection rate among people under 15 in aggregates}

For the dependent variable "rate of detection among those under 15 ," an association with the following socioeconomic factors was demonstrated: average number of inhabitants per house, the Gini coefficient, and the highest percentage of urban population ${ }^{(37)}$.

\section{DISCUSSION}

At the individual level, socioeconomic conditions were related to a higher incidence of leprosy, worsening of quality of life, poor adherence to treatment and evolution to physical impairment. In addition, they contributed to the prevalence of the disease among inside and outside the person's house contacts. The socioeconomic factors that were related to a greater individual risk were: schooling, unemployment, income less than a minimum wage, food shortage, non-permanent residence, houses made of wood or wattle and daub, number of rooms and people at home, collection of solid waste and existence electricity.

In the case of recurrence there is evidence that it is more likely to occur due to individual conditions, adult/elderly life stage, male gender, and multibacterial operational classification ${ }^{(38)}$.

Illiteracy and few years of study have been identified as risk factors for illness and evolution for physical impairment, since they make it difficult to recognize the clinical manifestations of the disease, access to the health system and the understanding of health education guidelines ${ }^{(11,39)}$. It is common for illiteracy and low schooling to occur more frequently among those with low socioeconomic conditions ${ }^{(13,40)}$.

There was a higher rate of unemployment, lower wages and the receipt of government financial aid among individuals with leprosy, especially those with physical impairment, contributing to a deterioration in the quality of life. Unemployment and low income are associated with monetary loss, employability due to limitations in the performance of professional activities by impaired individuals $s^{(7,39)}$, prejudice of employers, decreased social acceptance due to visible deformities, and social isolation of the patient after diagnosis ${ }^{(24)}$.

The experience of food shortages has increased the risk of illness and the occurrence of physical impairment. Low-income families may have fewer resources to obtain food of adequate nutritional value, resulting in food shortages and even hunger. Consequently, nutritional deficiencies increase susceptibility to infectious diseases ${ }^{(13)}$.

It is known that poor nutrition impairs cell-mediated immunity, which increases susceptibility to disease in individuals with subclinical infections ${ }^{(17)}$. Respiratory infections, diarrhea and malaria have been associated with malnutrition reported in the literature ${ }^{(41)}$.

Housing conditions also influenced the risk of infection, illness and recurrence of the disease. Poor housing conditions and a greater number of people in houses intensify exposure to the bacillus among home contacts of patients with leprosy ${ }^{(27)}$ and may even contribute to exogenous and recurrent reinfection ${ }^{(8)}$.

Lower home density contributed to the reduction of $M$. leprae infection among inside house contacts from anti-PGL1 seropositivity analysis ${ }^{(9)}$. The relation of insufficient or lack of access to basic sanitation, water supply, garbage collection and electric power supply with leprosy remains contradictory, with studies reporting a higher risk ${ }^{(14,27-28)}$ and others with no significant association ${ }^{(10)}$.

However, clusters of leprosy among neighboring houses are observed in areas with high population density, which bring together families with low socioeconomic status and do not have access to public services ${ }^{(42)}$.

The distance between houses and health facilities is indicated as a risk factor for the late diagnosis and the evolution for severe physical impairment ${ }^{(43)}$. Recurrence of this disease was more frequent among patients who used public transportation to arrive at the health facility, so factors associated with transportation difficulties reinforce the need for decentralization of leprosy control actions. Decentralized care facilitates access to health services and contributes to the timely diagnosis, adherence to treatment and greater equity in the assistance to cases of leprosy ${ }^{(8,44)}$.

At the ecological level, it was possible to verify that indicators related to low schooling, urbanization, lack of basic sanitation, high number of people per house and low Human Development 
Index (HDI), measured by longevity, income and education were associated with the occurrence of leprosy.

Before the strong relation between leprosy and unfavorable socioeconomic factors ${ }^{(32-33,35)}$, it is important to emphasize that the poverty condition does not lead to the transmissibility of the disease itself, but rather to the poverty condition with population densities in non-ventilated and places with lack of natural light, lack of adequate food to meet nutritional needs ${ }^{(17)}$, difficulty to access health services, among others.

Thus, once the material conditions necessary for human subsistence, related to food, housing, education, basic sanitation, environmental conditions, access to health services, among others $^{(33,35,37)}$ are not provided, they condition the occurrence of leprosy, when someone under these conditions lives with a sick person without proper treatment. In general, people affected by leprosy have low levels of schooling, which may lead to a lack of understanding about the disease, duration of treatment, perception of illness, and other health-related attitudes. Failure to recognize signs and symptoms of the disease in its initial stage leads to physical impairment and deformities, in addition to fueling the chain of transmission of this disease.

In the same train of thought, income is placed as a factor that reflects consumer power. When resources are insufficient for the acquisition of goods and services essential to human survival the chances of illness of the population multiply by the situation of vulnerability to which it is exposed. A study carried out in the state of Belo Horizonte shows higher rates of detection of leprosy in places of greater vulnerability ${ }^{(45)}$.

In studies of different levels of spatial aggregation, housing conditions are strongly related to the transmissibility of leprosy, both in the general population and in children; this is because the number of people living in a house with limited space and without healthy conditions, promotes the vulnerability of the environment and favors illness ${ }^{(46)}$. Children who share these spaces with people with no established diagnosis and appropriate treatment are at increased risk of falling ill.

Collective actions, such as the implementation of Family Health Strategy (Estratégia de Saúde da Família) services, alongside government poverty reduction programs such as the Child Benefit program, have shown positive impacts on epidemiological indicators of leprosy in Brazil ${ }^{(2,47)}$.
Therefore, actions that promote greater improvement in education and reduction of socioeconomic inequalities may increase the control of leprosy ${ }^{(1)}$.

\section{Study limitations}

Some limitations of this review should be considered: the restriction of the period of data coverage and the inclusion of articles available only in English, Portuguese and Spanish. Research restricted to some databases may have obscured available studies on less usual bases, despite the concern to explore diverse bases the choice may have directed the results. In addition, it is important to note that many surveys have used secondary data sources, which depend directly on the organization of local services and filling quality.

\section{Contributions to the Nursing, Health or Public Policy Sectors}

As a contribution, the review points out as strategies the coping of inequalities to the provision of health services capable of promoting equity of access and health care quality. The construction of innovative Nursing practices considering the context must be based on evidences. The adoption of the principle of equity is a decisive step in the elaboration of public policies that aim to reduce social and health inequalities.

\section{CONCLUSION}

When analyzing the association studies between leprosy and socioeconomic factors in different localities, differences were found in the results. This fact may be related to the characteristics of the population and/or level of aggregation of the studies.

It is concluded that leprosy suffers a great influence of the social context in which the patient is inserted, because the chances of exposing people to illness are the result of a set of not only individual aspects, but also of contexts or collective conditions. Thus, it is necessary to transcend the set of individual actions for a collective approach to health problems, considering the implementation of intersectoral actions to reduce social inequalities and improve living conditions. It is imperative to foster investments in social policies, in addition to train health professionals, given the importance of services of health care quality as minimizers of social inequities.

\section{REFERENCES}

1. Barreto ML, Teixeira MG, Bastos Fl, Ximenes AA, Barata RB, Rodrigues LC. Successes and failures in the control of infectious diseases in Brazil: social and environmental context, policies, interventions, and research needs. Lancet [Internet]. 2011 [cited 2017 Jul 27];47-60. Available from: http://www.thelancet.com/pdfs/journals/lancet/PIIS0140-6736(11)60202-X.pdf

2. Nery JS, Pereira SM, Rasella D, Penna MLF, Aquino R, Rodrigues LC, et al. Effect of the Brazilian conditional cash transfer and primary health care programs on the new case detection rate of leprosy. PLoS Negl Trop Dis [Internet]. 2014 [cited 2017 Jul 27];8(11):e3357. Available from: http://journals.plos.org/plosntds/article/file?id=10.1371/journal.pntd.0003357\&type=printable

3. Alfonso JL, Vich FA, Vilata JJ, de las Aguas JT. Factors contributing to the decline of leprosy in Spain in the second half of the twentieth century. Int J Lepr Outros Mycobact Dis [Internet]. 2005 [cited 2017 Jul 27];73(4):258-68. Available from: http://ila.ilsl.br/pdfs/v73n4a04.pdf

4. Miema A, Irgens LM, van Oortmarssen GJ, Richardus JH, Habbema JD. Disappearance of leprosy from Norway: an exploration of critical factors using an epidemiological modelling approach. Int J Epidemiol [Internet]. 2002 [cited 2017 Jul 27];31(5):991-1000. Available from: https://goo.gl/H72x7B 
5. Lanza FM, Lana FCF. O processo de trabalho em hanseníase: tecnologias e atuação da equipe de saúde da família. Texto Contexto Enferm [Internet]. 2011 [cited 2017 Jul 27];20(spe):238-246. Available from: http://www.scielo.br/pdf/tce/v20nspe/v20nspea30.pdf

6. Whittemore R, Knafl K. The integrative review: updated methodology. J Adv Nurs. 2005. Dec; 52(5): 546-53.

7. Diffey B, Vaz M, Soares MJ, Jacob AJ, Piers LS. The effect of leprosy-induced deformity on the nutritional status of index cases and their household members in rural South India: a socio-economic perspective. Eur J Clin Nutr [Internet]. 2000 [cited 2017 Jul 27];54(8):643-9. Available from: https://www.ncbi.nlm.nih.gov/pubmed/10951513

8. Ferreira SM, Ignotti E, Gamba MA. Factors associated to relapse of leprosy in Mato Grosso, Central-Western Brazil. Rev Saude Publica[Internet]. 2011 [cited 2017 Jul 27]; 45(4):756-64. Available from: http://www.scielo.br/pdf/rsp/v45n4/en_2587.pdf

9. Fabri ACOC. Prevalência de infecção pelo Mycobacterium leprae na população da microrregião de Almenara - [Dissertação] Belo Horizonte (MG): Universidade Federal de Minas Gerais. 2011

10. Hegazy AA, Abdel-Hamid IA, Ahmed el SF, Hammad SM, Hawas SA. Leprosy in a high-prevalence Egyptian village: epidemiology and risk factors. Int J Dermatol [Internet]. 2002 [cited 2017 Jul 27]; 41(10):681-6. Available from: https://onlinelibrary.wiley.com/doi/ abs/10.1046/j.1365-4362.2002.01602.x

11. Nardi SMT, Paschoal VD, Chiaravalloti-Neto F, Zanetta DMT. Leprosy-related disabilities after release from multidrug treatment: Prevalence and spatial distribution. Rev Saude Publica [Internet]. 2012 [cited 2017 Jul 27]; 46(6):969-77. Available from: http://www.scielo.br/pdf/rsp/ v46n6/en_ao4048.pdf

12. Araujo AERAE, Aquino DMC, Goulart IMB, Pereira SRF, Figueiredo IA, Serra HO, et al. Factors associated with neural alterations and physical disabilities in patients with leprosy in São Luis, State of Maranhão, Brazil. Rev Soc Bras Med Trop [Internet]. 2014 [cited 2017 Jul 27 ]; 47(4):490-7. Available from http://dx.doi.org/10.1590/0037-8682-0119-2014

13. Kerr-Pontes LR, Barreto ML, Evangelista CM, Rodrigues LC, Heukelbach J, Feldmeier H. Socioeconomic, environmental, and behavioural risk factors for leprosy in North-east Brazil: results of a case-control study. Int J Epidemiol [Internet]. 2006 [cited 2017 Jul 27]; 35(4):994-1000. Available from: https://www.ncbi.nlm.nih.gov/pubmed/16645029

14. Murto C, Ariza L, Alencar CH, Chichava OA, Oliveira AR, Kaplan C, et al. Migration among individuals with leprosy: a population-based study in central Brazil. Cad Saude Publica [Internet]. 2014 [cited 2017 Jul 27];30(3):487-501. Available from: http://www.scielo.br/pdf/csp/ v30n3/0102-311X-csp-30-3-0487.pdf

15. Santos DS, Duppre NC, Sales AM, Nery JAC, Sarno EN, Hacker MA. Kinship and Leprosy in the Contacts of Leprosy Patients: Cohort at the Souza Araújo Outpatient Clinic, Rio de Janeiro, RJ, 1987-2010. J Trop Med[Internet]. 2013 [cited 2017 Jul 27]; 2013: 596316. Available from: https://www.hindawi.com/journals/jtm/2013/596316/

16. Samuel P, Bushanam JDRS, Ebenezer M, Richard J. Impact of migration on new case detection rates in leprosy in Gudiyatham Taluk, Tamil Nadu, India. Indian J Lepr [Internet]. 2012 [cited 2017 Jul 27]; 84(4):307-16. Available from: https://www.ncbi.nlm.nih.gov/ pubmed/23720895

17. Feenstra SG, Nahar Q, Pahan D, Oskam L, Richardus JH. Recent food shortage is associated with leprosy disease in Bangladesh: a casecontrol study. PLoS NegI Trop Dis [Internet]. 2011 [cited 2017 Jul 27];5(5):e1029. Available from: https://journals.plos.org/plosntds/ article?id=10.1371/journal.pntd.0001029

18. Heukelbach J, Chichava OA, Oliveira AR, Häfner K, Walther F, Alencar CHM, et al. Interruption and defaulting of multidrug therapy against leprosy: population-based study in Brazil's Savannah Region. PLoS Negl Trop Dis [Internet]. 2011 [cited 2017 Jul 27];5(5):e1031. Available from: http://journals.plos.org/plosntds/article/file?id=10.1371/journal.pntd.0001031\&type=printable

19. Ribeiro GC. Fatores relacionados à prevalência de incapacidades físicas em Hanseníase na microrregião de Diamantina(MG)[Dissertação]. Belo Horizonte( MG): Universidade Federal de Minas Gerais. 2012.

20. Santos ASD, Castro DSd, Falqueto A. Fatores de risco para transmissão da Hanseníase. Rev Bras Enferm [Internet]. 2008 [cited 2017 Jul 27];61(esp):738-43. Available from: http://www.scielo.br/pdf/reben/v61nspe/a14v61esp.pdf

21. Wagenaar I, van Muiden L, Alam K, Bowers R, Hossain MA, Kispotta K, et al. Diet-related risk factors for leprosy: a case-control study. PLoS Negl Trop Dis [Internet]. 2015 [cited 2017 Jul 27]; 9(5):e0003766. Available from: https://www.ncbi.nlm.nih.gov/pmc/articles/ PMC4428634/

22. Mondal A, Kumar P, Das NK, Datta PK. A clinicodemographic study of lepra reaction in patients attending dermatology department of a tertiary care hospital in Eastern India. J Pakistan Assoc Dermatol [Internet]. 2015 [cited 2017 Jul 27];25(4):252-8. Available from: https:// www.researchgate.net/publication/296674120_A_clinicodemographic_study_of_lepra_reaction_in_patients_attending_dermatology_ department_of_a_tertiary_care_hospital_in_Eastern_India

23. Sanyal D, Gupta D, Mahapatra N, Samanta SK. A process report on physical and psychological determiners of social functioning in leprosy patients. Indian J Lepr [Internet]. 2011 [cited 2017 Jul 27]; 83(4):225-9. Available from: https://www.ncbi.nlm.nih.gov/pubmed/22783757

24. Costa MD, Terra FS, Costa RD, Lyon S, Costa AMDD, Antunes CMF. Assessment of quality of life of patients with leprosy reactional states treated in a dermatology reference center. An Bras Dermatol [Internet]. 2012 [cited 2017 Jul 27];87(1):26-35. Available from: http://www. scielo.br/pdf/abd/v87n1/v87n1a03.pdf

25. Kar S, Pal R, Bharati D. Understanding non-compliance with WHO-multidrug therapy among leprosy patients in Assam, India. J Neurosci Rural Pract [Internet]. 2010 [cited 2017 Jul 27];1(1):9-13. Available from: https://www.ncbi.nlm.nih.gov/pmc/articles/PMC3137843/ 
26. Kumar R, Singhasivanon P, Sherchand JB, Mahaisavariya P, Kaewkungwal J, Peerapakorn S, et al. Gender difference in socio-epidemiological factors for leprosy in the most hyper-endemic district of Nepal. Nepal Med Coll J [Internet]. 2004 [cited 2017 Jul 27];6(2):98-105. Available from: https://www.ncbi.nlm.nih.gov/pubmed/16295738

27. Murto C, Chammartin F, Schwarz K, Costa LM, Kaplan C, Heukelbach J. Patterns of migration and risks associated with leprosy among migrants in Maranhao, Brazil. PLoS Negl Trop Dis [Internet]. 2013 [cited 2017 Jul 27];7(9):e2422. Available from: https://journals.plos.org/ plosntds/article?id=10.1371/journal.pntd.0002422

28. Withington SG, Joha S, Baird D, Brink M, Brink J. Assessing socio-economic factors in relation to stigmatization, impairment status, and selection for socio-economic rehabilitation: A 1-year cohort of new leprosy cases in north Bangladesh. Lep rev [Internet]. 2003 [cited 2017 Jul 27];74(2):120-32. Available from: https://www.lepra.org.uk/platforms/lepra/files/lr/June03/06-120.pdf

29. Amaral EP. Análise espacial da hanseníase na microrregião de Almenara - Minas Gerais: relações entre a situação epidemiológica e as condições socioeconômicas [Dissertação] Belo Horizonte (MG): Universidade Federal de Minas Gerais. 2008.

30. Kerr-Pontes LR, Montenegro AC, Barreto ML, Werneck GL, Feldmeier H. Inequality and leprosy in Northeast Brazil: an ecological study. Int J Epidemiol [Internet]. 2004 [cited 2017 Jul 27]; 33(2):262-9. Available from: https://www.ncbi.nlm.nih.gov/pubmed/15082624

31. Queiroz JW, Dias GH, Nobre ML, Dias MCS, Araújo SF, Barbosa JD, et al. Geographic information systems and applied spatial statistics are efficient tools to study Hansen's disease (leprosy) and to determine areas of greater risk of disease. Am J Trop Med Hyg [Internet]. 2010 [cited 2017 Jul 27];82(2):306-14. Available from: https://www.ncbi.nlm.nih.gov/pubmed/20134009

32. Lana FCF, Davi RFL, Lanza FM, Amaral EP. Detecção da hanseníase e índice de desenvolvimento humano dos municípios de Minas Gerais, Brasil. Rev Eletrônica Enferm [Internet]. 2009 [cited 2017 Jul 27];11(3):539-44. Available from: https://www.fen.ufg.br/revista/v11/n3/pdf/ v11n3a10.pdf

33. Cury MR, Paschoal VD, Nardi SM, Chierotti AP, Rodrigues Junior AL, Chiaravalloti-Neto F. Spatial analysis of leprosy incidence and associated socioeconomic factors. Rev Saude Publica [Internet]. 2012 [cited 2017 Jul 27];46(1):110-8. Available from: http://www.scielo.br/pdf/rsp/ v46n1/3087.pdf

34. Imbiriba EN, Silva Neto AL, Souza WV, Pedrosa V, Cunha MG, Garnelo L. Social inequality, urban growth and leprosy in Manaus: a spatial approach. Rev Saude Publica [Internet]. 2009 [cited 2017 Jul 27];43(4):656-65. Available from: http://www.scielo.br/pdf/rsp/v43n4/en_842.pdf

35. Freitas LRS, Duarte EC, Garcia LP. Leprosy in Brazil and its association with characteristics of municipalities: ecological study, 2009-2011. Trop Med Int Health [Internet]. 2014 [cited 2017 Jul 27];19(10):1216-25. Available from: http://onlinelibrary.wiley.com/doi/10.1111/tmi.12362/epdf

36. Silva DR, Ignotti E, Souza-Santos R, Hacon Sde S. Hansen's disease, social conditions, and deforestation in the Brazilian Amazon.Rev Panam Salud Publica [Internet]. 2010 [cited 2017 Jul 27];27(4):268-75. Available from: https://www.ncbi.nlm.nih.gov/pubmed/20512229

37. Cabral-Miranda W, Chiaravalloti Neto F, Barrozo LV. Socio-economic and environmental effects influencing the development of leprosy in Bahia, north-eastern Brazil. Trop Med Int Health [Internet]. 2014 [cited 2017 Jul 27];19(12):1504-14. Available from: https://www.ncbi.nlm. nih.gov/pubmed/25244417

38. Araújo FCB, Souza CNP, Ramos EMLS, Braga RM. Aspectos associados à recidiva da hanseníase. Rev Bras Biom [Internet]. 2015 [cited 2017 Sep 26];33(1):42. Available from: http://jaguar.fcav.unesp.br/RME/fasciculos/v33/v33_n1/A4_Flavia_Cristiane.pdf

39. Guthi VR, Arepalli S, Ganapa P. Study of socio demographic factors among persons affected by leprosy in Kurnool division of Kurnool district, Andhra Pradesh, India. Int J Community Med Public Health [Internet]. 2016 [cited 2017 Sep 26];3(12):3548-55. Available from: https://www.scopemed.org/?mno=246302

40. Martins RJ, Carloni MEOG, Moimaz SAS, Garbin CAS, Garbin AJl. Sociodemographic and epidemiological profile of leprosy patients in an endemic region in Brazil Rev Soc Bras Med Trop [Internet]. 2016 [cited 2017 Sep 26];49(6):777-80. Available from: http://www.scielo.br/pdf/ rsbmt/v49n6/0037-8682-rsbmt-49-06-00777.pdf

41. Schaible UE, Kaufmann SHE. Malnutrition and infection: complex mechanisms and global impacts. PLoS Med [Internet]. 2007 [cited 2017 Jul 27]; 4(5):e115. Available from: http://dx.plos.org/10.1371/journal.pmed.0040115

42. Moura MLN, Dupnik KM, Sampaio GAA, Nóbrega PFC, Jeronimo AK, Nascimento-Filho JM, et al. Active Surveillance of Hansen's Disease (Leprosy): importance for case finding among extra-domiciliary contacts. PLoS Negl Trop Dis [Internet]. 2013 [cited 2017 Jul 27 ];7(3):e2093. Available from: https://www.ncbi.nlm.nih.gov/pmc/articles/PMC3597486/pdf/pntd.0002093.pdf

43. Muthuvel T, Govindarajulu S, Isaakidis P, Shewade HD, Rokade V, Singh R, Kamble S. "I Wasted 3 Years, Thinking It's Not a Problem": Patient and Health System Delays in Diagnosis of Leprosy in India: A Mixed-Methods Study. PLoS Negl Trop Dis [Internet]. 2017 [cited 2017 Sep 26]; 11(1): e0005192. Available from: https://www.ncbi.nlm.nih.gov/pubmed/28081131

44. Barbieri RR, Sales AM, Hacker MA, Nery JAC, Duppre NC, Machado AM, Moraes MO, Sarno EN. Impact of a Reference Center on Leprosy Control under a Decentralized Public Health Care Policy in Brazil. PLoS Negl Trop Dis [Internet]. 2017 [cited 2017 Sep 26];10(10):e0005059. Available from: https://www.ncbi.nlm.nih.gov/pubmed/27732610

45. Monteiro LD, Mota RMS, Martins-Melo FR, Alencar CH, Heukelbach J. Social determinants of leprosy in a hyperendemic State in North Brazil. Rev Saude Publica [Internet]. 2017 [cited 2017 Sep 26];51:70. Available from: https://www.ncbi.nlm.nih.gov/pubmed/28746575

46. Rodrigues RN, Niitsuma ENA, Bueno IC, Baquero OS, Jardim CCG, Lana FCF. Leprosy and health vulnerability in Belo Horizonte, (MG). Rev Min Enferm [Internet]. 2017 [cited 2017 Jun 13];21:e-997. Available from: http://www.reme.org.br/artigo/detalhes/1133

47. Penna MLF, Grossi MADF, Penna GO. Country profile: leprosy in Brazil. Lepr Rev [Internet]. 2013 [cited 2017 Jul 27];84(4):308-15. Available from: https://www.lepra.org.uk/platforms/lepra/files/lr/dec13/lep308-315.pdf 